\title{
National survey of patient specific IMRT quality assurance in China
}

\author{
Yuxi Pan ${ }^{1}$, Ruijie Yang ${ }^{1 *}$, Shuming Zhang ${ }^{1}$, Jiaqi $\mathrm{Li}^{1}$, Jianrong Dai ${ }^{2}$, Junjie Wang ${ }^{1}$ and Jing Cai ${ }^{3}$
}

\begin{abstract}
Background: To analyze and present the China's national survey on patient-specific IMRT quality assurance (QA).

Methods: A national survey was conducted in all radiotherapy centers in China to collect comprehensive information on status of IMRT QA practice, including machine, technique, equipment, issues and suggestions.

Results: Four hundred and three centers responded to this survey, accounting for $56.92 \%$ of all the centers implementing IMRT in China. The total number of medical physicists and the total number of patients treated with IMRT annually in these centers was 1599 and 305,000 respectively. All centers implemented measurement-based verification. Point dose verification and 2D dose verification was implemented in 331 and 399 centers, respectively. Three hundred forty-eight centers had 2D arrays, and 52 centers had detector devices designed to measure VMAT beams. EPID and film were used in 78 and 70 centers, respectively. Seventeen and 20 centers used log file and 3D DVH analysis, respectively. One hundred sixty-eight centers performed measurement-based verification not for each patient based on different selection criteria. The techniques and methods varied significantly in both point dose and dose distribution verification, from evaluation metrics, criteria, tolerance limit, and steps to check failed IMRT QA plans. Major issues identified in this survey were the limited resources of physicists, QA devices, and linacs.

Conclusions: IMRT QA was implemented in all the surveyed centers. The practice of IMRT QA varied significantly between centers. An increase in personnel, QA devices and linacs is highly desired. National standard, guideline, regulation and training programs are urgently needed in China for consistent and effective implementation of IMRT QA.
\end{abstract}

Keywords: IMRT, Patient-specific QA, National survey

\section{Background}

Patient-specific intensity-modulated radiotherapy (IMRT) quality assurance (QA) is critically important to the successful implementation of IMRT. It ensures correct machine-sided delivery of the prescribed dose by checking the accuracy of dose calculation, plan transfer, and treatment delivery. It is strongly recommended as part of the IMRT clinical process by the professional societies such as the American Association of Physicists in Medicine (AAPM), the American Society for Radiation Oncology (ASTRO), the American College of Radiology (ACR), and the Netherlands Commission on Radiation Dosimetry [1-5]. Guidelines and recommendations for IMRT and VMAT QA have also been published, clearly stating

\footnotetext{
* Correspondence: ruijyang@yahoo.com

${ }^{1}$ Department of Radiation Oncology, Peking University Third Hospital, 49th North Garden Road, Haidian District, Beijing 100191, People's Republic of China

Full list of author information is available at the end of the article
}

that patient-specific IMRT QA is necessary to ensure patient safety. However, none of these publications addresses the issue of how patient-specific IMRT QA should be performed explicitly [6-9]. Recently, AAPM Task Group (TG) 218 reported recommendation on measurement and analysis methods, and tolerance limits for patient-specific IMRT QA [10].

In the past decade, IMRT has been widely adopted in China due to the pressing clinical needs of advanced radiotherapy technologies for treating a booming population of cancer patients nationwide. IMRT has been implemented in more than 700 centers in China, and volumetricmodulated arc therapy (VMAT) has been introduced in more than 110 centers in a much faster pace than that of the fixed gantry IMRT [11]. Despite these advances, to date, there is no national standard or practice guideline on patient-specific IMRT QA in China.

(c) The Author(s). 2019 Open Access This article is distributed under the terms of the Creative Commons Attribution 4.0 International License (http://creativecommons.org/licenses/by/4.0/), which permits unrestricted use, distribution, and reproduction in any medium, provided you give appropriate credit to the original author(s) and the source, provide a link to the Creative Commons license, and indicate if changes were made. The Creative Commons Public Domain Dedication waiver (http://creativecommons.org/publicdomain/zero/1.0/) applies to the data made available in this article, unless otherwise stated. 
A Work Group on Commissioning and Patient Specific IMRT QA, responsible for drafting the national standard and guideline of IMRT QA, was established under the guidance of the Radiation Oncology Quality Control Committee, the National Quality Control Center of Cancer Theranostics, and the National Health Commission of the People's Republic of China in June 2017. This Work Group conducted a national survey in September 2017 on the implementation of patient-specific IMRT QA and multi-center validation test of IMRT QA. Information of IMRT uptake, equipment, delivery techniques, QA devices, QA techniques and methods were collected in the survey from radiotherapy centers in China. The purpose of the survey is to collect comprehensive information on and identify key issues of current IMRT QA practice in China for establishing national guidelines and national multi-center validation tests for implementing patientspecific IMRT QA in China. The Work Group will make recommendations based on the survey results on patient-specific IMRT QA methods, tools and devices, time and frequency, comparison approach, evaluation metrics, criteria and tolerance limits, and data interpretation. These recommendations will be implemented through professional guidelines and government policy to improve IMRT QA practice in China to ensure treatment fidelity and patient safety. In this paper, we report the findings of China's national survey on patient-specific IMRT QA.

\section{Methods}

This survey was conducted in form of questionnaires October 2017 to December 2017. A questionnaire was sent out to the members of the IMRT QA Work Group in each province, autonomous region and municipality directly under the Central Government of China (excluding Hong Kong and Macao Special Administrative Regions and Taiwan province), and then was distributed to radiotherapy centers that have implemented IMRT. Once completed, the questionnaires were collected via email and verified for each item. In case the questionnaire response was found to be substandard, a list of identified problems will be complied and sent back to the respondents via email for clarifications.

In this survey, IMRT was defined as the inverselyplanned intensity-modulated radiotherapy techniques. All linac-based IMRT delivery techniques, static and rotating gantry (including TomoTherapy), were included in the survey. Survey questions covered the general information about the radiotherapy centers, medical physicists, IMRT delivery techniques, equipment, patient characteristics, patient-specific IMRT QA details, problems, and suggestions. The patient-specific IMRT QA details included dose verification tools and methods, normalization, dose threshold, data interpretation, tolerance and action limits, method of checking failed IMRT QA plans, Multi-leaf Collimator (MLC) QA, et al.

Descriptive statistics were performed for all variables using SPSS (IBM Corp, Armonk, NY, USA) for Windows, version 22 .

\section{Results \\ Responding centers and patients treated}

Four hundred and three centers responded to this survey, accounting for $56.92 \%$ of the centers that are currently practicing IMRT in China. Among the 403 centers, 152 centers have also implemented VMAT. A wide range of IMRT experience was reported, ranging from greater than ten years to less than three months. The 403 responding centers included 41 cancer hospitals and 362 general hospitals; or 100 academic hospitals and 303 non-academic hospitals. Three hundred five thousand patients were treated with IMRT per year in the responding centers (Fig. 1). The most commonly treated sites were lung, breast, cervical, nasopharyngeal, esophageal, and rectal cancer.

\section{Medical physicists}

In all responding centers, medical physicists are reported to be responsible for the IMRT QA program. Radiotherapists and clinical engineers are also involved in IMRT QA under the supervision of medical physicists in 133 centers and 27 centers, respectively. Regardless of the QA performer, checking and approving of the IMRT QA results are performed by medical physicists. There are a total of 1599 medical physicists in the 403 responding centers (Fig. 2). The number of IMRT patients per physicist is shown in Fig. 3. More than 100 IMRT patients were treated per physicist in 272 centers.

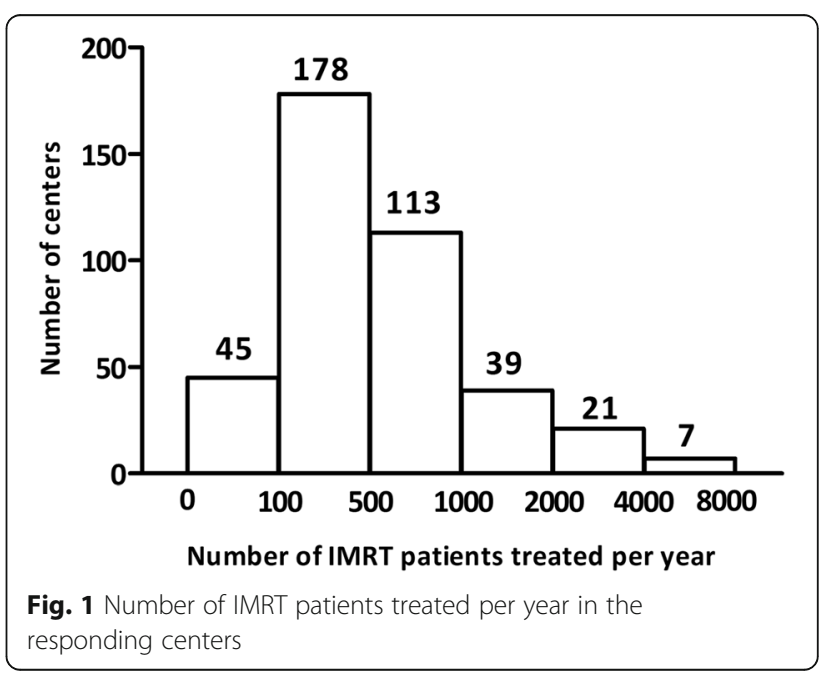




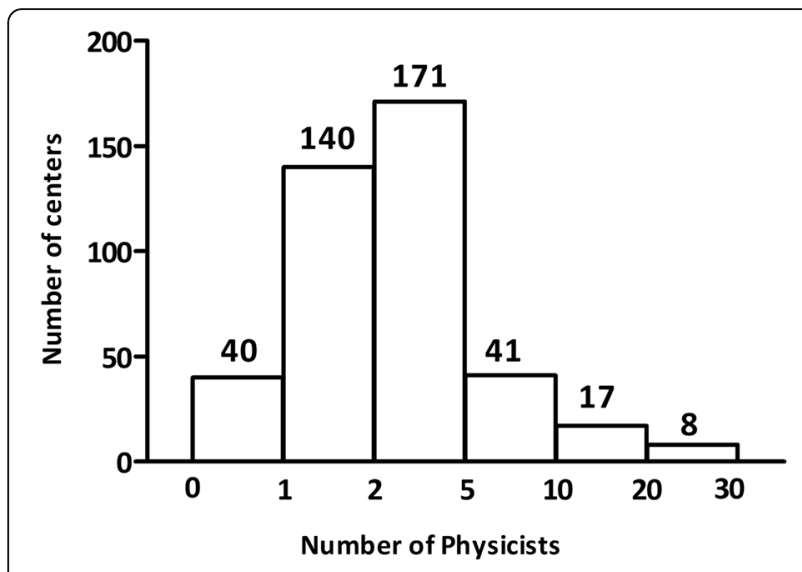

Fig. 2 Number of physicists in the responding centers

\section{Linacs and TPS}

Figure 4 shows the statistics of linear accelerators and treatment planning systems (TPS) used for IMRT. There are a total of 655 linacs and 819 TPS workstations in the responding centers. Some centers used linacs from more than one manufacturer. The number of centers that has $1,2,3,4$, and $\geq 5$ linacs is $277,92,18,13$, and 17 , respectively. The number of centers that has $1,2,3,4$, and $\geq 5$ TPSs is $257,71,32,10$ and 33 , respectively. The number of IMRT patients treated per linac is shown in Fig. 5. In more than half of the centers, the number of IMRT patients treated per linac was greater than 300 .

\section{Verification techniques and methodologies}

Table 1 summarizes the verification techniques and methodologies used for IMRT QA. All responding centers used measurement-based verification method. Three hundred thirty one centers used point dose verification, of those 4 centers used point dose verification only without dose distribution verification. Ion chambers with different sizes (range: $0.01 \mathrm{cc}$ to $0.6 \mathrm{cc}$ ) were used for point dose verification. For fixed gantry IMRT QA using

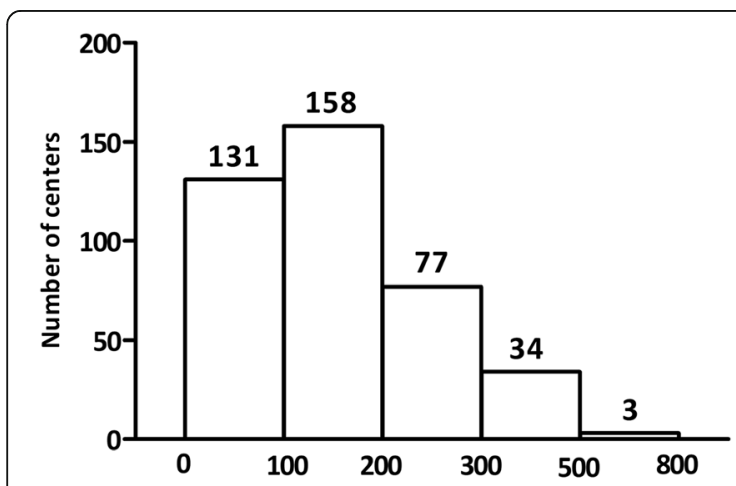

Number of IMRT patients treated per year/Number of physicists

Fig. 3 Number of IMRT patients treated per physicist in the responding centers

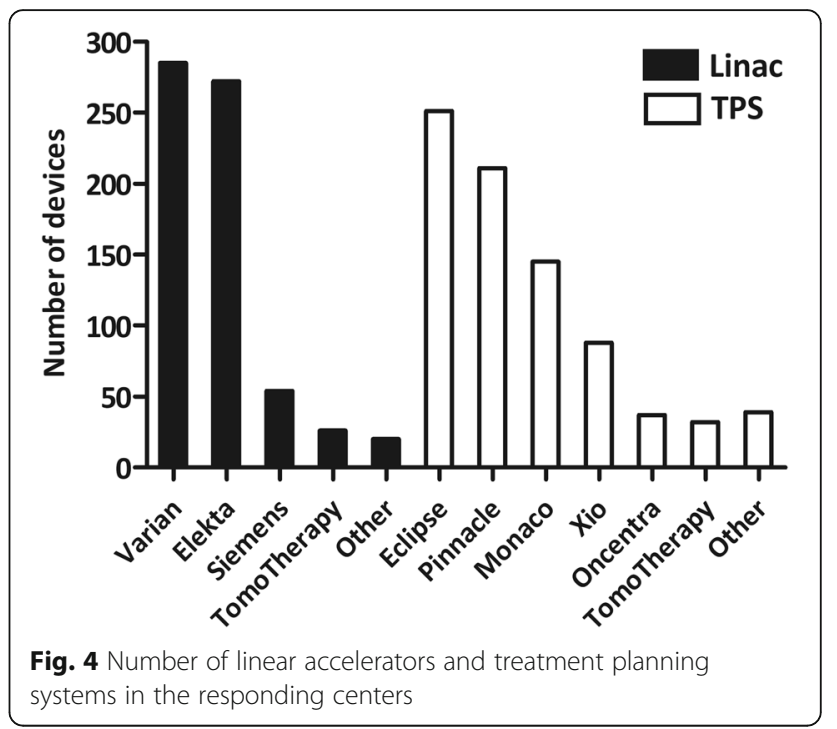

2D array devices, 107 centers used perpendicular fieldby-field (PFF) verification to improve efficiency when the IMRT QA results were out-of-tolerance using the perpendicular composite (PC) method. All the responding centers that have implemented VMAT $(n=152)$ applied true composite (TC) method for VMAT QA using Delta4, ArcCheck, Octavious with dedicated phantom, film, Compass or EPID, etc.

\section{Selection criteria of measurement-based QA}

235 (58.3\%) centers performed measurement-based IMRT QA for all of their IMRT patients, while 168 centers $(41.7 \%)$ only performed measurement-based IMRT QA for selected patients. The selection criteria varied between centers and included a wide range of factors such as tumor site, plan complexity, prescription dose, fractionation, normal tissue tolerances, treatment delivery technique, intent of treatment, availability of treatment machine, verification tools, physicist time, patient's

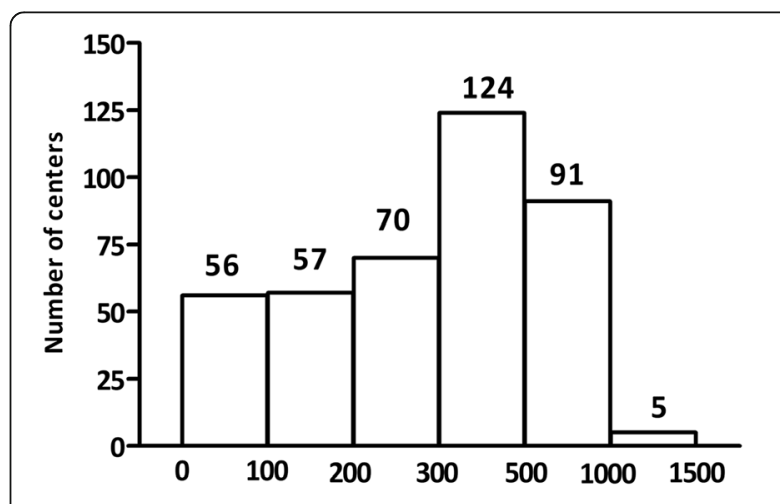

Number of IMRT patients treated per year/Number of linacs

Fig. 5 Number of IMRT patients treated per linac in the responding centers 
Table 1 Verification techniques and methodologies for patient specific IMRT QA in China

\begin{tabular}{|c|c|c|c|c|c|}
\hline \multirow{7}{*}{$\begin{array}{l}\text { Items } \\
\text { Techniques }\end{array}$} & & & & Number & Percentage (\%) \\
\hline & Point dose & Tools & Ion chamber & 331 & 82.1 \\
\hline & 2D dose & Tools & 2D diode or chamber arrays & 348 & 86.4 \\
\hline & & & EPID & 78 & 19.4 \\
\hline & & & Film + diode or ionization chamber arrays & 58 & 14.4 \\
\hline & & & Film & 12 & 3.0 \\
\hline & 3D dose & Tools & ArcCheck or Delta 4 & 52 & 12.9 \\
\hline \multirow[t]{38}{*}{ Methodologies } & Point dose & Location of ion chamber & Isocenter & 198 & 49.1 \\
\hline & & & Maximum dose point & 67 & 16.6 \\
\hline & & & Uniform high dose region & 39 & 9.7 \\
\hline & & & Isocenter or a uniform high dose region & 42 & 10.4 \\
\hline & & & $5 \mathrm{~cm}$ for $6 \mathrm{MV}, 10 \mathrm{~cm}$ for $10 \mathrm{MV}$ plans & 29 & 7.2 \\
\hline & & Measurement value & Mean dose to ion chamber volume & 171 & 42.4 \\
\hline & & & Point dose (effective measurement point) & 172 & 42.7 \\
\hline & & Tolerance limits & $2 \%$ & 3 & 0.7 \\
\hline & & & $3 \%$ & 356 & 88.3 \\
\hline & & & $4 \%$ & 1 & 0.2 \\
\hline & & & $5 \%$ & 21 & 5.2 \\
\hline & & & Other & 19 & 4.7 \\
\hline & & & No response & 17 & 4.2 \\
\hline & & Action limits & $2 \%$ & 35 & 8.2 \\
\hline & & & $3 \%$ & 154 & 38.2 \\
\hline & & & $5 \%$ & 78 & 19.4 \\
\hline & & & $10 \%$ & 1 & 0.2 \\
\hline & & & Other & 20 & 5.0 \\
\hline & & & No response & 161 & 40 \\
\hline & $2 \mathrm{D}, 3 \mathrm{D}$ dose & Delivery methods & Perpendicular field-by-field (PFF) & 190 & 47.1 \\
\hline & & & Perpendicular composite (PC) & 258 & 64.0 \\
\hline & & & True composite (TC) & 110 & 27.3 \\
\hline & & & PFF after PC failure & 107 & 26.6 \\
\hline & & Orientation of film/array & Coronal & 340 & 84.4 \\
\hline & & & Sagittal & 35 & 8.7 \\
\hline & & & Transverse & 41 & 10.2 \\
\hline & & Absolute dose calibration & Before each IMRT QA session & 115 & 28.5 \\
\hline & & & Weekly & 84 & 20.8 \\
\hline & & & Monthly & 128 & 31.8 \\
\hline & & & Every 3 months to one year & 70 & 17.4 \\
\hline & & & Never & 3 & 0.7 \\
\hline & & Grid size & $1 \mathrm{~mm}$ & 39 & 9.7 \\
\hline & & & $2 \mathrm{~mm}$ & 155 & 38.5 \\
\hline & & & $2.5 \mathrm{~mm}$ & 50 & 12.4 \\
\hline & & & $3 \mathrm{~mm}$ & 190 & 47.1 \\
\hline & & & $4 \mathrm{~mm}$ & 67 & 16.6 \\
\hline & & & $5 \mathrm{~mm}$ & 2 & 0.5 \\
\hline & & & Varied with TPS, delivery techniques & 75 & 18.6 \\
\hline
\end{tabular}


Table 1 Verification techniques and methodologies for patient specific IMRT QA in China (Continued)

\begin{tabular}{|c|c|c|c|c|}
\hline Items & & & Number & Percentage (\%) \\
\hline & Reference distribution & Measured dose & 205 & 50.9 \\
\hline & & Calculated dose & 282 & 70.0 \\
\hline & Dose algorithm & Pencil beam & 108 & 26.8 \\
\hline & & Convolution/and superposition & 278 & 69.0 \\
\hline & & Monte Carlo & 140 & 34.7 \\
\hline & Evaluation metrics & Dose difference (DD) at multiple points & 168 & 41.7 \\
\hline & & Distance-to-agreement (DTA), & 155 & 38.5 \\
\hline & & Gamma pass rate & 353 & 87.6 \\
\hline & & Profiles or isodose distributions & 157 & 39.0 \\
\hline & & Anatomy-based 3D dose distributions and DVHs & 20 & 5.0 \\
\hline & Tolerance limits & $90 \%$ & 38 & 9.4 \\
\hline & & $95 \%$ & 293 & 72.7 \\
\hline & & $93 \%$ & 1 & 0.2 \\
\hline & & No response & 85 & 21.1 \\
\hline & Action limits & $90 \%$ & 35 & 8.7 \\
\hline & & $95 \%$ & 262 & 65.0 \\
\hline & & $80 \%$ & 2 & 0.5 \\
\hline & & No response & 118 & 29.3 \\
\hline & Gamma criteria & $2 \% \mathrm{DD}$ & 35 & 8.7 \\
\hline & & $3 \% \mathrm{DD}$ & 305 & 75.7 \\
\hline & & $4 \% \mathrm{DD}$ & 17 & 4.2 \\
\hline & & $5 \% \mathrm{DD}$ & 57 & 14.1 \\
\hline & & $1 \mathrm{~mm}$ DTA & 8 & 2.0 \\
\hline & & $2 \mathrm{~mm}$ DTA & 50 & 12.4 \\
\hline & & $3 \mathrm{~mm}$ DTA & 300 & 74.7 \\
\hline & & $4 \mathrm{~mm}$ DTA & 15 & 3.7 \\
\hline & & $5 \mathrm{~mm}$ DTA & 1 & 0.2 \\
\hline & Normalization point & Maximum dose point & 207 & 51.4 \\
\hline & & Isocenter & 166 & 41.2 \\
\hline & & Other points in the high dose plateau region & 80 & 19.9 \\
\hline & Normalization modes & Global normalization & 306 & 75.9 \\
\hline & & Local normalization & 78 & 19.4 \\
\hline & Dose analysis modes & Absolute & 225 & 55.8 \\
\hline & & Relative & 235 & 58.3 \\
\hline & & Both & 85 & 21.1 \\
\hline & Dose thresholds & $10 \%$ & 286 & 71.0 \\
\hline & & $20 \%$ & 33 & 8.2 \\
\hline & & $5 \%$ or $15 \%$ & 38 & 9.4 \\
\hline Reasons and actions & Reasons for failed QA & Plan being too highly modulated & 287 & 71.2 \\
\hline & & Dose measurement point in a high gradient region & 232 & 57.6 \\
\hline & & Inaccurate phantom set up & 195 & 48.4 \\
\hline & & MLC positioning uncertainty & 201 & 49.9 \\
\hline & Actions for failed QA & Checking the verification plan & 296 & 73.4 \\
\hline & & Checking the VS, TPS and delivery system & 353 & 87.6 \\
\hline
\end{tabular}


Table 1 Verification techniques and methodologies for patient specific IMRT QA in China (Continued)

\begin{tabular}{|c|c|c|c|c|}
\hline Items & & & Number & Percentage (\%) \\
\hline & & Re-measure or design verification plan again & 317 & 78.7 \\
\hline & & Previous plan verification & 151 & 37.5 \\
\hline & & Communicated with physicians & 146 & 36.2 \\
\hline & & Re-plan & 199 & 49.4 \\
\hline \multirow[t]{5}{*}{ MLC QA } & Frequency & Monthly & 242 & 60.0 \\
\hline & & Weekly & 131 & 32.5 \\
\hline & & Daily & 66 & 16.4 \\
\hline & & Every season, half year or one year & 53 & 13.2 \\
\hline & & Never & 33 & 8.2 \\
\hline \multirow[t]{2}{*}{ Audits and clinical trial } & Type & External audits or inter-institution comparison & 168 & 41.7 \\
\hline & & Clinical trial credential & 13 & 3.2 \\
\hline \multirow[t]{4}{*}{ Issues of IMRT QA } & Type & Lack of physicists & 174 & 43.2 \\
\hline & & Lack of time & 230 & 57.1 \\
\hline & & Lack of QA devices & 196 & 48.6 \\
\hline & & Lack of linacs & 182 & 45.2 \\
\hline
\end{tabular}

economic status, reimbursement, physician and/or patient's preference, etc. Most of these centers performed patient-specific IMRT QA for all of their IMRT patients during the first year of IMRT implementation (or for the first 100 IMRT patients), after which they randomly selected patients for IMRT QA with a sampling rate ranging from 20 to $60 \%$.

For patients that measurement-based IMRT QA were not performed, 16 centers performed calculation-based verification, but majority of the centers did not perform any other type of verification. All centers performed measurement-based IMRT QA for hypo-fractionation radiotherapy, SBRT and SRS plans.

\section{Device calibration, reference distribution and dose calculation}

The frequency of absolute dose calibration for diode/ion chamber arrays varied between centers. Three centers had never performed absolute dose calibration after commissioning. Measured dose was used as reference in 205 centers, calculated dose was used as reference in 282 centers, and interpolation of measured dose was used as reference in 195 centers. Pencil beam, convolution/and superposition and Monte Carlo based dose calculation algorithms were used in 108, 278 and 140 centers, respectively. Grid size of $1,2,2.5,3,4$ and $5 \mathrm{~mm}$ was used for dose calculation in TPS in 39, 155, 50, 190, 67 and 2 centers. 1-4 mm was used in 75 centers based on different treatment planning system, delivery technique, or target size.

\section{Evaluation metrics, tolerances and action limits}

Different evaluation metrics, tolerance and action limits were used for point dose verification and $2 \mathrm{D}$ dose verification. Seventy seven centers evaluated the concordance between the calculated and the measured dose distributions with different metrics and criteria in different dose gradient regions.

The gamma criteria implemented for evaluating IMRT QA varied between centers. Dose difference (DD) of 2-5\% and dose to agreement (DTA) of $1-5 \mathrm{~mm}$ was used in these centers. The most commonly used DD/DTA value for gamma criteria was $3 \% / 3 \mathrm{~mm}$. For normalization methods, 207 centers used maximum dose point as the normalization point, 166 centers used isocenter and 80 centers used other points in the high dose plateau region. Global/local normalization was used in 306 and 78 centers, respectively. Dose analysis mode and threshold were also different between centers.

\section{Causes and actions for failed IMRT QA results}

The most common causes for failed IMRT QA cases were over modulation, point dose measurement in a high dose gradient region, incorrect QA phantom setup, and MLC leaf position uncertainty. Other causes included TPS beam modeling error, QA planning error, small field or narrow long field, QA device error, linac output error, IMRT QA analysis error, and laser issues.

Most centers reported difficulties in analyzing root causes and providing solutions for failed IMRT QA cases. The methods of investigating failed IMRT QA included checking the verification plan, checking the verification system, planning system, and delivery system, repeating measurement, and repeating the entire IMRT QA verification. Three hundred forty nine centers checked the treatment machine consistency, verification system and treatment planning system performance, 195 
centers edited the IMRT plan based on the results of IMRT QA, and 144 centers discussed with the radiation oncologist to make a clinical decision.

\section{MLC QA, external audits, multiple institution comparison, and clinical trials}

The frequency of MLC QA varied between centers. Thirty three centers did not perform MLC QA. The MLC tests, including leaf calibration and position accuracy, were performed using film, EPID, 2D array, graph papers or log files. One hundred sixty eight centers reported participation in external IMRT audits or inter-institution comparison, and 13 centers were credentialed for clinical trials with IMRT.

\section{Issues and suggestions}

This survey revealed a significant issue of limited resources in physicist staffing, time, QA device, and treatment machine. In some centers, IMRT QA was voluntarily performed by medical physicists without salary compensation for working overtime. There was no reimbursement for IMRT QA in majority of the centers. One hundred thirty seven centers performed IMRT QA before the first treatment for hypo-fractionated treatments, SRS and SBRT. Two hundred fifty one centers performed IMRT QA during the first three fractions for conventional fractionated treatment. IMRT QA was performed during working hour in day time in 124 centers, in the evening in 177 centers, and on weekend in 283 centers.

The most concerning issues on IMRT QA devices and techniques included the optimal size of ion chamber for point dose measurement, the accuracy and comparability of various techniques and devices with different hardware and software. IMRT QA was largely considered time-consuming, complex and cumbersome in this survey. Easy-to-use devices with high resolution and high efficiency are highly desired. The clinical significance of IMRT QA results was unclear and it is difficult to appreciate for current 2D, phantom-based IMRT QA techniques. There was a lack of 3D anatomy-based IMRT QA devices and techniques.

\section{Discussion}

A national survey on patient-specific IMRT QA has been successfully conducted in China, including 56.92\% of the radiotherapy centers that are currently practicing IMRT in China. Overall, the survey results showed that significant variations exist in the implementation of patient-specific IMRT QA among radiotherapy centers, including techniques, equipment, evaluation criteria, etc. This finding reflects the great need of regulations in China for IMRT practice accreditation, certification, audit, examination and monitoring. Technical guidance, support and cooperation, and training programs should be continually implemented to improve the IMRT QA practice nationally, including training programs from QA device and linac manufacturers. The survey results also implies the urgent need to establish medical physicist profession in China.

It is revealed in this survey that burden of IMRT QA is considerably high for medical physicists in China. According to a framework published by American Society for Radiation Oncology (ASTRO) [12], the required number of physicists and dosimetrists for IMRT,IGRT,SRS,TBI and SBRT treatment equals to the patient number multiplied by the coefficient 0.008 and 0.005 , respectively. In China, physicist and dosimetrist are not separated and collectively referred to as physicist. Thus, 1.3 physicists(/dosimetrists) are needed for every 100 patients. However, this survey showed that more than 100 IMRT patients were treated per physicist in 272 centers (67\%), reflecting the serious workload problem for medical physicist in China. In addition, in more than half of the centers, the number of IMRT patients treated per linac was larger than 300 . Considering that there are also many other patients treated in one linac, such as patients treated with 3D-CRT, SBRT etc., implying that the machine is fully occupied for patient treatment during working hours and physicists can only do IMRT QA in the evening or during weekends in most of the centers. To address this significant challenge of limited machine time for IMRT QA, some centers chose the strategy of performing measurement-based IMRT QA for selected patients only after a few years of practicing IMRT.

The survey yielded comprehensive data on current practice of IMRT QA in China, including techniques, equipment, manpower, reimbursement model, etc., providing the evidence foundation for the Work Group on Commissioning and Patient Specific IMRT QA to develop China's national guidelines for implementing patient-specific IMRT QA. Based on the survey results, the Work Group has developed a series of recommendations for national guidelines for IMRT QA practice in China. Representative recommendations for dose verification and action response to IMRT QA failure are shown below:

\section{Dose verification}

For point dose verification, leakage current should be corrected when small volume ion chambers are used in point dose verification $[8,13]$. The ion chamber with adequate spatial resolution should be selected and placed in a plateau dose region, considering of the dose gradient and positioning errors. In general, the dose gradient across the ion chamber for homogeneous dose distributions should be less than $5 \%$ of the mean dose to the chamber. For SBRTISRS, it should be as close as possible to the criteria. The calculated dose to the chamber volume, instead of dose to the effective measurement point or 
middle of the chamber active volume should be compared with the measured dose.

For planar dose verification, if the angular dependence of 2D array is negligible or can be corrected accurately, the TC measurements can be used. Otherwise, the measurements should be performed using PFF method due to anisotropic dose response of the array detectors [14-16]. The PC method should not be used due to the possibility of masking delivery errors. No significant correlation was observed between PFF 3\%/3 mm DTA and the actual 3D dose differences [17-20]. A confidence limit difference of 12.4 and $7 \%$ for TC and PFF was noted in the TG-119 report, respectively [3]. So, tools for patient anatomy-based $3 \mathrm{D}$ verification and specially designed VMAT QA are highly desirable [21-25].

For gamma index analysis, the evaluated dose distribution should have the same or higher resolution than the reference distribution [26]. For the 282 centers that used calculated dose as the reference distribution, the comparison accuracy was compromised if no interpolation of measured dose distribution was used. When measured dose distribution is used as reference, small calculation grid size should be used. Dose calculation grid size larger than $3 \mathrm{~mm}$ is not appropriate for IMRT QA.

Dose difference, DTA, dose profiles and isodose distribution should be reviewed in addition to the gamma pass rate. Furthermore, not only the failure percentage, but also the maximum, average gamma value, and gamma distribution should be reviewed [11]. It is difficult to establish the acceptance limits for IMRT QA because different delivery systems, planning systems, and verification devices are used [27, 28]. Analyzing gamma pass rate with different dose difference/DTA criteria is useful to find the sources and judge the impact of discrepancies. Stricter criteria of $3 \% / 2 \mathrm{~mm}$, even $2 \% / 2 \mathrm{~mm}$ should be used, as the experience and confidence increase in IMRT QA. Some centers used locally defined limits varying with cancer site and plan complexity [29]. 10\% action limit for point dose verification, $5 \mathrm{~mm}$ DTA limits, and $80 \%$ action limit for gamma analysis is not acceptable. The European Society for Radiotherapy and Oncology (ESTRO) recommended tolerance and action limits of 3 and 5\% for ion chamber measurements [30]. AAPM recommended that tolerance and action limits should be within $\leq 2 \%$ and $\leq 3 \%$, respectively [11]. Planar dose verification using a $2 \mathrm{D}$ array with the detector spacing of $7 \mathrm{~mm}$ could not detect MLC leaf position errors smaller than $2 \mathrm{~mm}$, with $3 \% / 3 \mathrm{~mm}$ criteria and a $90 \%$ gamma passing rate [31]. The average deviations in $\mathrm{D}_{95 \%}$ of target, $\mathrm{D}_{0.1 \mathrm{cc}}$ of the spinal cord reached 8 and $12 \%$, respectively, in complex head and neck plans for systematic leaf position errors of $1 \mathrm{~mm}$ [32]. Therefore, tighter tolerances should be used together with accelerator and MLC QA [33, 34].
In addition, absolute dose mode should be used for IMRT QA analysis because considerable differences may go undetected using relative dose mode. The absolute dose calibration of the ion chamber or diode arrays should be performed before each measurement, in order to rule out the influence of detector response and accelerator output variation. Global normalization should be used due to its clinical relevance. The normalization point should be placed in a high dose, low gradient region, often the maximum dose point, not necessarily isocenter of the plan, especially when the isocenter is located in the low dose or high gradient region [35].

\section{Action response to IMRT QA failure}

If IMRT QA failed, medical physicist should systematically review the dose difference, DTA, gamma index, isodose distribution, dose profile, structure specific dose distribution and DVH when available, to determine if the dose deviations are clinically acceptable. A comprehensive root causes analysis should be performed to determine the reasons for these discrepancies and find the solution to them. It may be necessary to check the clinical plan, QA plan, QA device, setup, and/or measure with a different measurement device or different geometry. Medical physicists should understand the characteristics and performance of their QA tool, implementation details, and test its accuracy. If the modulation of the failed plan is much more complex than usual, planning with less complex intensity patterns should be considered. If the gamma passing rate is systematically lower than the recommended action limits, then the dose differences should be thoroughly reviewed, using local normalization and tighter criteria, to find subtle regional discrepancies.

The IMRT workflow should be thoroughly investigated, including the TPS beam modeling and commissioning, QA planning, QA device and linac performance testing, and end-to-end tests. Gamma passing rates should be tracked among patients for the same sites, to differentiate if the errors are specific for a treatment site, or delivery equipment. In addition, patientspecific verification QA for previous cases, the multiple center comparison or independent validation tests can also be performed to help identify the sources of errors.

The survey also revealed issues that need to be further investigated and discussed. For example, tools such as EPID and log file that can simplify the set-up, measurement or analysis of IMRT QA should be encouraged [36]. While there are controversies on the value and methods of patient-specific IMRT QA [37-40], especially whether computation can replace measurements, a significant increase is expected in calculation-based verification. For plans that the measurement-based IMRT 
QA was not performed, calculation-based verification should be considered, together with systematic QAs of linac and TPS [41]. Furthermore, reimbursement of IMRT QA should be addressed and made known to physicians, department directors and hospitals, as well as to the administration and public.

\section{Conclusions}

A national survey on patient-specific IMRT QA was successfully conducted in China. Patient-specific IMRT QA is implemented in all surveyed radiotherapy centers, but the practice varied significantly between centers. The survey shows that IMRT QA is a significant burden to IMRT practice in China, largely due to the limited resources in manpower, equipment and machine time. National standard and guideline, regulation and training programs for IMRT QA are urgently needed in China to ensure effective implementation of IMRT in high quality consistently.

\section{Abbreviations}

AAPM: The American Association of Physicists in Medicine; ACR: The American College of Radiology; ASTRO: The American Society for Radiation Oncology; ESTRO: The European Society for Radiotherapy and Oncology; IMRT: Intensity-modulated radiation therapy; MLC: Multi-leaf Collimator; PC: Perpendicular composite; PFF: Perpendicular field-by-field; QA: Quality assurance; TC: True composite

\section{Acknowledgements}

This work was supported by the Work Group on Commissioning and Patient Specific QA of IMRT, Radiation Oncology Quality Control Committee, National Quality Control Center of Cancer Theranostics, National Health Commission of the People's Republic of China. The authors would like to thank Xiaowu Deng, Jie Qiu, Qin Li, Mingxuan Jia, Xiance Jin, Yongming Liu, Dingjie Li, Fushan Zhai, Feng Xiao, Yanling Bai, Xiaofen Xing, Fu Jin, Pei Wang, Zhenyu Zhai, Qingguo Fu, Xiaobo Li, Huidong Wang, Xiaoping Wang et al. for supporting and coordinating in collecting the survey questionnaire. The authors would like to thank the medical physicists in the responding hospitals for taking the time to complete the survey.

\section{Funding}

This study was supported by National natural Science Foundation of China [Grant numbers: 81071237, 81372420].

\section{Availability of data and materials}

The datasets used and/or analyzed during the current study are available from the corresponding author on reasonable request.

\section{Authors' contributions}

RY designed this survey and made major contributions in writing the manuscript and collecting the data. YP analyzed the data and was a major writer. SM and JL collected and summarized the survey data. JD made contributions in collecting the questionnaires. JW and JC provide suggestions on how to analyze the results, JC also contributed in writing and revising the manuscript. All authors read and approved the final manuscript.

\section{Ethics approval and consent to participate}

Not applicable.

\section{Consent for publication}

Not applicable.

\section{Competing interests}

The authors declare that they have no competing interests.

\section{Publisher's Note}

Springer Nature remains neutral with regard to jurisdictional claims in published maps and institutional affiliations.

\section{Author details}

'Department of Radiation Oncology, Peking University Third Hospital, 49th North Garden Road, Haidian District, Beijing 100191, People's Republic of China. ${ }^{2}$ Department of Radiation Oncology, Chinese Academy of Medical Science Cancer Institute, 17 Panjiayuan Nanli, Beijing, People's Republic of China. ${ }^{3}$ Department of Health Technology and Informatics, The Hongkong Polytechnic University, Hongkong, People's Republic of China.

Received: 15 December 2018 Accepted: 8 April 2019

Published online: 25 April 2019

\section{References}

1. Ezzell GA, Galvin JM, Low D, et al. Guidance document on delivery, treatment planning, and clinical implementation of IMRT: report of the IMRT subcommittee of the AAPM radiation therapy committee. Med Phys. 2003;30:2089-115.

2. Ezzell GA, Burmeister JW, Dogan N, et al. IMRT commissioning: multiple institution planning and dosimetry comparisons, a report from AAPM task group 119. Med Phys. 2009;36:5359-73.

3. Moran JM, Dempsey M, Eisbruch A, et al. Safety considerations for IMRT: executive summary. Med Phys. 2011;38:5067.

4. Hartford AC, Galvin JM, Beyer DC, et al. American College of Radiology (ACR) and American Society for Radiation Oncology (ASTRO) practice guideline for intensity-modulated radiation therapy (IMRT). Am J Clin Oncol. 2012:35:612-7.

5. Mans A, Schuring D, Arends MP, et al. The NCS code of practice for the quality assurance and control for volumetric modulated arc therapy. Phys Med Biol. 2016;61:7221-35

6. Fraass B, Doppke $\mathrm{K}$, Hunt $\mathrm{M}$, et al. American Association of Physicists in Medicine radiation therapy committee task group 53: quality assurance for clinical radiotherapy treatment planning. Med Phys. 1998;25:1773-829.

7. Das IJ, Cheng CW, Watts RJ, et al. Accelerator beam data commissioning equipment and procedures: report of the TG-106 of the Therapy Physics Committee of the AAPM. Med Phys. 2008;35(9):4186-215

8. Low DA, Moran JM, Dempsey JF. Dosimetry tools and techniques for IMRT. Med Phys. 2011;38:1313-38.

9. Jacamin DJ, Bredfeldt JS, Frigo SP. Implementation of the validation testing in MPPG 5.a "Commissioning and QA of treatment planning dose calculations-megavoltage photon and electron beams". J Appl Clin Med Phys. 2017;18:115-27.

10. Miften M, Olch A, Mihailidis D, et al. Tolerance limits and methodologies for IMRT measurement-based verification QA: recommendations of AAPM task group no. 218. Med Phys. 2018;45:e53-83.

11. Lang JY, Wang P, Wu DK, et al. An investigation of the basic situation of radiotherapy in mainland China in 2015. Chin J Radiat Oncol. 2016;25:541-5.

12. ASTRO. Safety is no accident. A framework for quality radiation oncology and care, 2012.

13. Leybovich LB, Sethi A, Dogan N. Comparison of ionization chambers of various volumes for IMRT absolute dose verification. Med Phys. 2003:30:119-23.

14. Jursinic PA, Sharma R, Reuter J. MapCHECK used for rotational IMRT measurements: step-and-shoot, TomoTherapy, RapidArc. Med Phys. 2010;37:2837-46

15. Van Esch A, Clermont C, Devillers M, et al. On-line quality assurance of rotational radiotherapy treatment delivery by means of a $2 \mathrm{D}$ ion chamber array and the Octavius phantom. Med Phys. 2007;34:3825-37.

16. Feygelman $\mathrm{V}$, Forster K, Opp D. Evaluation of a biplanar diode array dosimeter for quality assurance of step-and-shoot IMRT. J Appl Clin Med Phys. 2009;10:3080.

17. Kruse JJ. On the insensitivity of single field planar dosimetry to IMRT inaccuracies. Med Phys. 2010;37:2516-24.

18. Nelms BE, Zhen H, Tome WA. Per-beam, planar IMRT QA passing rates do not predict clinically relevant patient dose errors. Med Phys. 2011;38:1037.

19. Stasi M, Bresciani S, Miranti A, et al. Pretreatment patient-specific IMRT quality assurance: a correlation study between gamma index and patient clinical dose volume histogram. Med Phys. 2012;39:7626-34. 
20. Carrasco P, Jornet N, Latorre A, et al. 3D DVH-based metric analysis versus per-beam planar analysis in IMRT pretreatment verification. Med Phys. 2012; 39:5040-9.

21. Letourneau D, Publicover J, Kozelka J, et al. Novel dosimetric phantom for quality assurance of volumetric modulated arc therapy. Med Phys. 2009;36:1813-21.

22. Yan G, Lu B, Kozelka J, et al. Calibration of a novel four dimensional diode array. Med Phys. 2010;37:108-15.

23. Feygelman V, Zhang G, Stevens $C$, et al. Evaluation of a new VMAT QA device, or the " $\mathrm{X}$ " and "O" array geometries. J Appl Clin Med Phys. 2011;12:3346.

24. Kozelka J, Robinson J, Nelms B, et al. Optimizing the accuracy of a helical diode array dosimeter: a comprehensive calibration methodology coupled with a novel virtual inclinometer. Med Phys. 2011;38:5021-32.

25. Yang RJ, Wang JJ, Xu F, et al. Electronic portal imaging device for Dosimetric verification of intensity-modulated radiation therapy. Chin J Radio Med Prot. 2013;33:41-4.

26. Low DA, Dempsey JF. Evaluation of the gamma dose distribution comparison method. Med Phys. 2003;30:2455-64.

27. Ibbott GS, Followill DS, Molineu HA, et al. Challenges in credentialing institutions and participants in advanced technology multi-institutional clinical trials. Int J Radiat Oncol Biol Phys. 2008:71:S71-5.

28. Das IJ, Cheng CW, Chopra KL, et al. Intensity-modulated radiation therapy dose prescription, recording, and delivery: patterns of variability among institutions and treatment planning systems. J Natl Cancer Inst. 2008;100:300-7.

29. Yang R, Zhang X, Liu L, et al. Analysis of 2010 patient-specific intensitymodulated radiation therapy dosimetric verification results. Chin J Radiol Med Prot. 2016;12:917-21.

30. Alber M, Broggi C, De Wagter C, et al. Guidelines for the verification of IMRT. ESTRO booklet, 2008

31. Yan G, Liu C, Simon TA, et al. On the sensitivity of patient-specific IMRT QA to MLC positioning errors. J Appl Clin Med Phys. 2009;10:2915.

32. Mu G, Ludlum E, Xia P. Impact of MLC leaf position errors on simple and complex IMRT plans for head and neck cancer. Phys Med Biol. 2008;53:77-88.

33. Zhen $H$, Nelms BE, Tome WA. Moving from gamma passing rates to patient DVH-based QA metrics in pretreatment dose QA. Med Phys. 2011:38:5477-89.

34. Nelms BE, Chan MF, Jarry G, et al. Evaluating IMRT and VMAT dose accuracy: practical examples of failure to detect systematic errors when applying a commonly used metric and action levels. Med Phys. 2013;40:1117221-11172215.

35. Bailey DW, Nelms BE, Attwood K, et al. Statistical variability and confidence intervals for planar dose QA pass rates. Med Phys. 2011;38:6053.

36. Yang R, Wang J, Xu F, et al. The electronic portal imaging device for dosimetric verification of intensity-modulated radiation therapy. Chin $J$ Radiol Med Prot. 2013:33:55-7.

37. Pawlicki T, Yoo S, Court LE, et al. Moving from IMRT QA measurements toward independent computer calculations using control charts. Radiother Oncol. 2008;89:330-7.

38. Smith JC, Dieterich S, Orton CG. It is STILL necessary to validate each individual IMRT treatment plan with dosimetric measurements before delivery. Med Phys. 2011;38:553-5.

39. Siochi RAC, Molineu A, Orton CG. Patient-specific QA for IMRT should be performed using software rather than hardware methods. Med Phys. 2013; 40:0706011-3.

40. Kruse JJ, Mayo CS. Comment on "catching errors with patient-specific pretreatment machine log file analysis". Pract Radiat Oncol. 2012;3:91-2

41. Yang RJ, Wang JJ, Zhang XL, et al. Dosimetric effect of multi-leaf collimator transmission on intensity-modulated radiation therapy. Chin J Radiat Oncol. 2013;22:157-60

Ready to submit your research? Choose BMC and benefit from:

- fast, convenient online submission

- thorough peer review by experienced researchers in your field

- rapid publication on acceptance

- support for research data, including large and complex data types

- gold Open Access which fosters wider collaboration and increased citations

- maximum visibility for your research: over $100 \mathrm{M}$ website views per year

At $\mathrm{BMC}$, research is always in progress.

Learn more biomedcentral.com/submissions 